Check for updates

Cite this: RSC Adv., 2018, 8, 7980

Received 23rd October 2017 Accepted 5th February 2018

DOI: $10.1039 / c 7 r a 11702 g$

rsc.li/rsc-advances

\section{Saturation and beating of acoustic phonon oscillations excited near the exciton resonance of strained polar $\mathrm{ZnO} / \mathrm{Zn}_{0.8} \mathrm{Mg}_{0.2} \mathrm{O}$ multiple quantum wells}

\author{
Wei-Rein Liu, (DD *a Ja-Hon Lin, ${ }^{\text {b }}$ Jyun-Sian Chen, ${ }^{\mathrm{b}}$ Hsin-Ming Cheng, ${ }^{\mathrm{c}}$ Sheng-Jie Li, ${ }^{\mathrm{b}}$ \\ Hou-Ren Chen, ${ }^{d}$ Chia-Hung Hsuad and Wen-Feng Hsieh ${ }^{d}$
}

\begin{abstract}
Saturation and beating of coherent acoustic phonon (CAP) oscillations were observed and attributed to the screening of a built-in electric field with increasing pump power using degenerate pump-probe measurements near the exciton resonance of polar $\mathrm{ZnO} / \mathrm{Zn}_{0.8} \mathrm{Mg}_{0.2} \mathrm{O}$ multiple quantum wells (MQWs). After purifying the CAP signals by using an empirical mode decomposition, we found not only that the CAP amplitude follows the trend of the band gap renormalization (BGR) and shows saturation at high pump power, but also that the CAP oscillation period coincides with that of the MQWs, consistent with the XRD and TEM results. An additional low-frequency oscillation modifying the CAP signal is revealed due to the negative change in refractive index caused by BGR as the pump power increases.
\end{abstract}

\section{Introduction}

Over the past few decades, coherent phonon oscillations with frequencies in the terahertz range have attracted considerable attention because of their practical applications in phonon spectroscopy, nano-ultrasonic imaging ${ }^{1,2}$ and ultrafast modulation of optics and electronics. The mechanisms which generate coherent phonon oscillation have been studied extensively. Several mechanisms have been proposed to explain those oscillations, including impulsive stimulated Raman scattering (ISRS) for transparent media ${ }^{4,5}$ and the most frequently cited displacive excitation of coherent phonons (DECP) mechanism or recently resonant ISRS in opaque materials. ${ }^{3}$ The generation of photo-induced coherent acoustic phonon (CAP) oscillations is strongly correlated with electronphonon and photon-phonon coupling mechanisms, ${ }^{6}$ which provide new physical insights into the properties of acoustic phonons and their interactions with surrounding carriers in complex and correlated systems.

By changing the period width of multiple-quantum wells (MQWs) providing efficient confinement of carriers or superlattices (SLS), the CAP oscillation frequency can be tuned. The

${ }^{a}$ National Synchrotron Radiation Research Center, Hsinchu 30076, Taiwan. E-mail: liu.weirein@nsrrc.org.tw

${ }^{b}$ Department of Electro-Optical Engineering, National Taipei University of Technology, Taipei 10608, Taiwan.E-mail: jhlin@ntut.edu.tw

${ }^{c}$ Material and Chemical Research Laboratories, Industrial Technology Research Institute, Hsinchu 31040, Taiwan

${ }^{d}$ Department of Photonics, Institute of Electro-Optical Engineering, National Chiao Tung University, Hsinchu 30010, Taiwan oscillation frequency $\nu$ is related to the SL period $\Lambda_{\mathrm{SL}}$ and the sound velocity $c_{\mathrm{s}}$ in the medium by the formula: $\nu=c_{\mathrm{s}} / \Lambda_{\mathrm{SL}}{ }^{7}$ Due to the artificial periodicity the folding of the acoustic branches induced additional optical phonon-like modes in the artificial lattice, termed as zone folded longitudinal acoustic phonons, with non-zero frequencies at the Brillouin zone center $(q \cong 0)$. This enables the observation of higher frequency zone-folded CAP oscillations. Different from the CAPs generated in a GaAs material system, originating from the deformation potential coupling, ${ }^{8-10}$ large amplitude CAP oscillations in InGaN MQWs were observed due to the electron-phonon interaction being enhanced by the strong piezoelectric coupling mechanism..$^{11,12}$

As a counterpart to $\mathrm{GaN}$, because of the stronger excitonic oscillator strength and the larger exciton binding energy ( $\sim 60$ $\mathrm{meV}$ ) at room temperature (RT), wide-band-gap ZnO semiconductors have become promising candidates for various UV photonic devices, e.g., LEDs ${ }^{13}$ and RT polariton lasers. ${ }^{14}$ Due to their strong piezoelectricity, they can also be used as sensors and transducers. Recently, the ultrafast carrier dynamics of polar $^{15,16}$ and non-polar ${ }^{17,18} \mathrm{ZnO}$ epitaxial films have been investigated through optical pump-probe transient differential reflection (TDR) spectroscopy. Furthermore, the generation of CAPs in a $600 \mathrm{~nm} c$-ZnO film on silicon through Brillouin scattering had also been demonstrated when the excited photon energy was set below the exciton resonance of the sample. ${ }^{19}$

$\mathrm{ZnO} / \mathrm{ZnMgO} \mathrm{MQW}$ structures enhance the localization of carriers and photons in the well regions and so increase the exciton binding energy and interaction strength, nevertheless, being grown along the $c$-axis leads to an internal electric field being exerted across the quantum well which causes 
a quantum-confined Stark effect (QCSE). ${ }^{20}$ This internal electric field is attributed to spontaneous and piezoelectric polarizations, resulting from lattice mismatches between the well and barrier materials. However, to the best of our knowledge, an investigation into the correlation of the THz CAP oscillations with the carrier dynamics in strained polar $\mathrm{ZnO} / \mathrm{ZnMgO} \mathrm{MQWS}$ has never been reported.

In this study, the structural properties of ten-period $\mathrm{ZnO} /$ $\mathrm{Zn}_{0.8} \mathrm{Mg}_{0.2} \mathrm{O} \mathrm{MQWs}$ (ZnO/ZnMgO MQWs) on (0001) sapphire substrates grown using pulsed laser deposition (PLD) were thoroughly examined using X-ray diffraction (XRD) and transmission electron microscopy (TEM). By performing degenerate pump-probe transient differential transmission (TDT) and reflection (TDR) spectroscopy, the sub-THz CAP and carrier dynamics in $c$-axis $\mathrm{ZnO} / \mathrm{ZnMgO}$ MQWs were investigated using a photo-excitation energy set between the band edge and the exciton resonance of the $\mathrm{ZnO}$ well layers. The mechanism of CAP generation is attributed to the internal electric field being perturbed by the photocarrier screening field. The CAP signal was analyzed by using the empirical mode decomposition, ${ }^{21,22}$ and the noise and the low-frequency oscillation signal can be filtered out. The results indicate that the CAP oscillation is strongly correlated with band gap renormalization (BGR).

\section{Experimental section}

\section{Sample preparation}

Ten-period $\mathrm{ZnO} / \mathrm{ZnMgO} \mathrm{MQWs}$ were grown on double-sidepolished (0001) sapphire substrates using PLD. A KrF excimer laser with a wavelength of $248 \mathrm{~nm}$ was focused on commercial hot-pressed stoichiometric $\mathrm{ZnO}(4 \mathrm{~N}), \mathrm{Zn}_{0.97} \mathrm{Mg}_{0.03} \mathrm{O}(3 \mathrm{~N})$ and $\mathrm{Zn}_{0.8} \mathrm{Mg}_{0.2} \mathrm{O}(3 \mathrm{~N})$ targets with a pulse energy density of $\sim 2-3 \mathrm{~J}$ $\mathrm{cm}^{-2}$. To minimize the effect of magnesium diffusion into the well layers by the thermal driving force at high growth temperatures, we adopted a temperature-gradient control method, in which the growth temperature of the barriers was set lower than that of the well layers. Prior to fabrication of the MQWs, a $23.5 \mathrm{~nm}$-thick $\mathrm{Zn}_{0.97} \mathrm{Mg}_{0.03} \mathrm{O}$ buffer layer and a $39 \mathrm{~nm}$ thick $\mathrm{Zn}_{0.8} \mathrm{Mg}_{0.2} \mathrm{O}$ barrier were grown on the $c$-plane sapphire substrate. Then, alternating $\mathrm{ZnO}$ wells and $\mathrm{Zn}_{0.8} \mathrm{Mg}_{0.2} \mathrm{O}$ barriers were deposited.

\section{Identification of structural and optical properties}

XRD measurements were performed with a nine-circle diffractometer with an incident wavelength of $1.0331 \AA$ at the IU22 undulator beamline TPS-09A of the Taiwan Photon Source at the National Synchrotron Radiation Research Center. Two pairs of slits between the sample and the $\mathrm{LaCl}_{3}$ scintillation detector yield a resolution of over $3.2 \times 10^{-3} \AA^{-1}$. A cross-sectional TEM sample with a thickness of approximately $90 \pm 10 \mathrm{~nm}$ was prepared using a focused ion beam. In addition, the microstructure and interface qualities of the samples were analyzed using scanning transmission electron microscopy (STEM) performed with a JEOL $2100 \mathrm{~F}$ microscope equipped with an energydispersive X-ray detector operating at $200 \mathrm{keV}$. RT photoluminescence (PL) was measured under the excitation of a He-
Cd laser with a wavelength of $325 \mathrm{~nm}$ using a monochromator (iHR 550, Horiba Inc.). The transmittance and reflectance spectra were also measured using a white light source (Ocean Optics DH-2000-BAL) and a spectrometer (iHR 320, Horiba Inc.).

\section{Degenerate pump-probe measurement}

Fig. 1a illustrates the experimental setup for TDT and TDR (inset) measurements using the degenerate pump-probe technique. A mode-locked Ti:sapphire laser (Tsunami, Spectral Physics Inc.) equipped with a frequency doubler (Model 3980, Spectral Physics Inc.) producing linearly polarized UV pulsed light with $80 \mathrm{MHz}$ repetition rate and 105 fs pulse width was used.

By passing the beam through a beam splitter and a half-wave $(\lambda / 2)$ plate, two orthogonal polarized beams were used as the pump beam (red solid line) and the probe beam (purple dashed line), respectively. After passing through a convergent lens, these two beams were focused simultaneously onto the same location on the sample at an angle of $12^{\circ}$. The horizontal polarized pump beam has an average power which has been set from $4 \mathrm{~mW}$ to $16 \mathrm{~mW}$ or 4 to $16 \mu \mathrm{J} \mathrm{cm}^{-2}$ and the vertical polarized probe beam has a fixed power of approximately 0.5 $\mathrm{mW}$ or $0.5 \mu \mathrm{J} \mathrm{cm}^{-2}$. The relative time delay between the pump and probe beams was controlled with a high-resolution translation stage. To filter out the residual pump beam, an aperture and a linear polarizer were placed in front of the detector. For the reflection scheme (upper right in Fig. 1a), the probe beam reflected from the sample was divided and collimated by a beam splitter and a mirror. Finally, the transmitted and reflected probe beams were collected by the detector. In order to
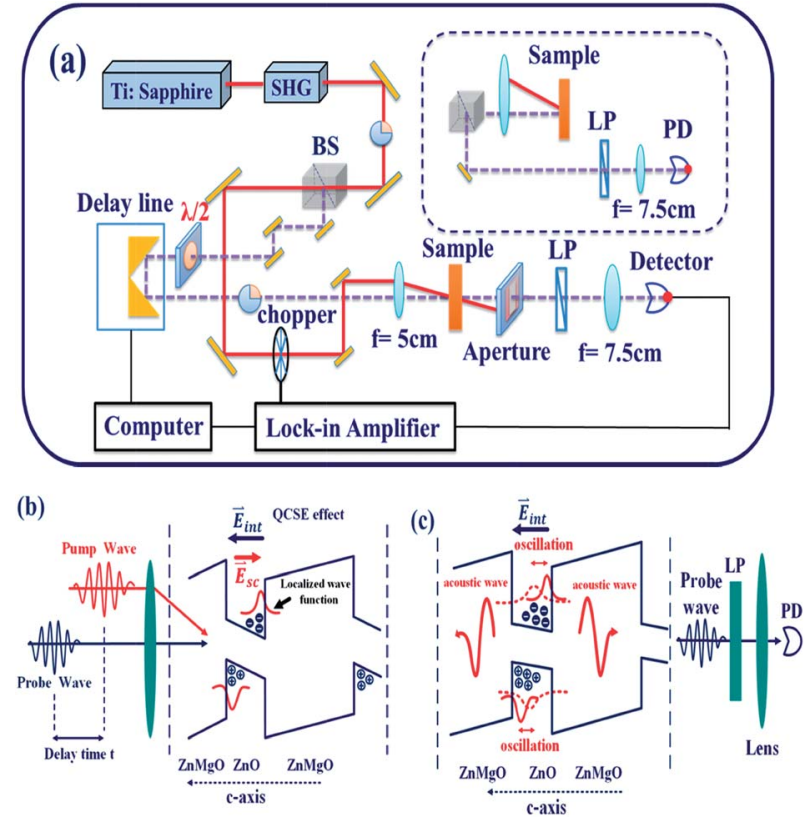

Fig. 1 Schematic diagram of (a) the experimental setup for the TDT and TDR degenerate pump-probe measurements and the (b) generation and (c) detection of acoustic waves in $\mathrm{ZnO} / \mathrm{ZnMgO}$ MQWs. 
efficiently increase the signal-to-noise ratio of the received signal, we adopted a lock-in amplifier incorporating a mechanical chopper in the path of the pump beam.

The CAP generation and detection mechanisms from the $\mathrm{ZnO} / \mathrm{ZnMgO} \mathrm{MQW}$ are illustrated in Fig. $1 \mathrm{~b}$ and c, respectively. $\mathrm{ZnO}$ consists of alternating layers of $\mathrm{Zn}$ cations and $\mathrm{O}$ anions along the $c$-axis. This, along with the piezoelectric field induced by the strain at the quantum well interfaces due to lattice mismatch between the $\mathrm{ZnO}$ and $\mathrm{ZnMgO}$ layers, induces a net spontaneous dipole and piezoelectric fields along the $c$-axis. The net internal electric field, $E_{\text {int }}$, causes band tilting, reducing the exciton oscillator strength through separation of the electron and hole wave functions, and increasing the exciton lifetime. These are referred to as the quantum-confined Stark effects (QCSEs). ${ }^{20}$ After the absorption of the femtosecond UV pumppulse by the $c$-axis ZnO wells in the MQWs, the periodic distributions of photo-excited carriers, with the electrons and holes localized near the conduction and valence bands, respectively, produce a screening field $E_{\mathrm{sc}}$ (red arrow in Fig. 1c). Then the internal electric field is perturbed by $E_{\mathrm{sc}}$ to generate the displacive CAP oscillation, ${ }^{\mathbf{1 1}}$ which propagates in the $+c$ and $-c$ directions. Therefore, the reflected and transmitted probe beams are modulated by the CAP oscillation, and the oscillation is observable in both the TDR and TDT traces.

\section{Results and discussion}

Fig. 2a shows the X-ray reflectivity of the $\mathrm{ZnO} / \mathrm{ZnMgO}$ MQWs on (0001) sapphire substrates. The reflectivity curve exhibits
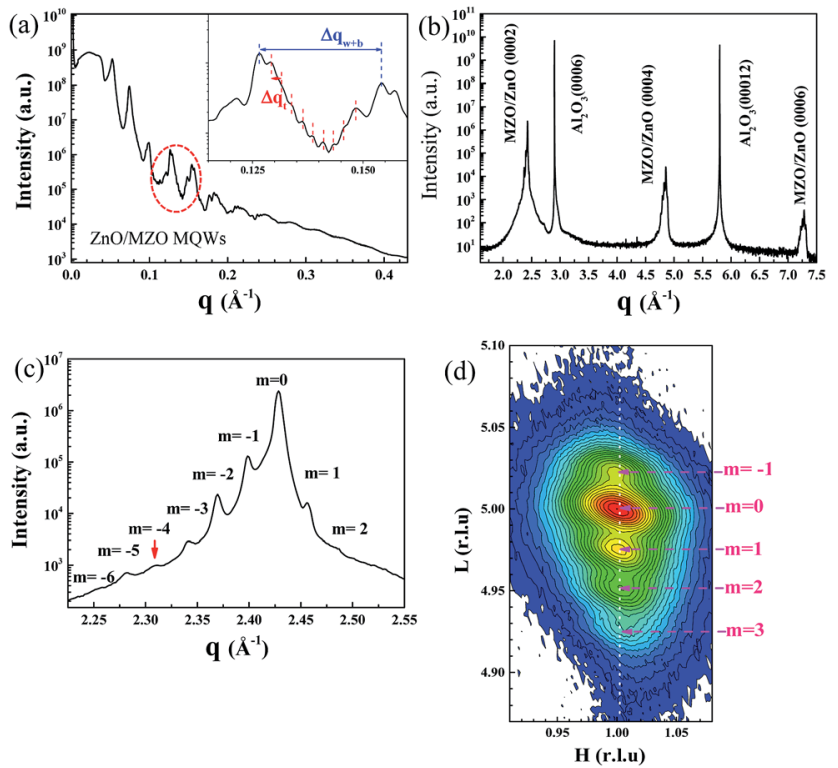

Fig. 2 (a) Reflectivity curve of the $\mathrm{ZnO} / \mathrm{ZnMgO} M Q W s$ deposited on a (0001) sapphire substrate. The inset shows the enlarged reflectivity curve of the circled region. (b) XRD radial scan along the surface normal of the $\mathrm{ZnO} / \mathrm{ZnMgO} \mathrm{MQWs}$ grown on a (0001) sapphire substrate. (c) Enlarged XRD radial scan around the $\mathrm{ZnO} / \mathrm{ZnMgO} M Q W$ (0002) diffraction peak. (d) XRD RSM at the (1015) ZnO/ZnMgO MQW reflection. a typical shape with Bragg peaks separated by Kisseig fringes. The Bragg peaks are correlated with a MQW period of $\sim 22.76 \mathrm{~nm}$, determined from $\Delta q_{\mathrm{w}+\mathrm{b}}=0.276 \mathrm{~nm}^{-1}$ (inset of Fig. 2a). The fringes are qualitatively ascribed to the interference between X-rays scattered by the difference in electron density between the well and barrier layers at the interface regime, which is evidence for the vertical periodicity of the composition modulation. Furthermore, the appearance of satellite peaks among the regime of Bragg peaks is ascribed to the difference in electron density between the substrate and the film; the period of the fringes reflects the films' partial thickness of $\sim 182 \mathrm{~nm}$, calculated from $\Delta q_{\mathrm{t}}=0.03445 \mathrm{~nm}^{-1}$. This result represents the eight-period thickness of the MQWS because the total thickness of the films is beyond the thickness detection limit of X-ray reflectivity. The presence of these clear oscillations indicates that the surface and the interface are well correlated, and smooth enough to produce these oscillations.

A typical radial scan along the surface normal of the $\mathrm{ZnO} /$ ZnMgO MQW sample is also illustrated in Fig. 2b. The observation of only MQW (0002), (0004) and (0006) reflections, together with the $\mathrm{Al}_{2} \mathrm{O}_{3}$ (0006) and (00012) reflections, demonstrates that the $\mathrm{ZnO} / \mathrm{ZnMgO} \mathrm{MQW}$ structure is $c$-plane oriented with its [0001] axis parallel to the $\mathrm{Al}_{2} \mathrm{O}_{3}$ [0001] direction. Furthermore, it is worth mentioning that the appearance of well-resolved satellite peaks of the $5^{\text {th }}$ order around the (0002) peak (Fig. 2c) arises from interference between the X-ray waves reflected from the sample structure. This is an indication of the high crystalline quality of the MQW structure, because both interface imperfection and compositional inhomogeneity would decrease the phase coherence and suppress the intensity of the satellite peaks. However, the $4^{\text {th }}$-order satellite peak is missing in the XRD pattern. From a scattering perspective, ${ }^{23}$ the MQW structure can be considered as a one-dimensional artificial crystal that is comprised of a number of unit cells with a period $\Lambda_{\mathrm{MQw}}$. Each unit cell consists of two sublayers, i.e., a barrier and a well, with thicknesses $\Lambda_{\text {barrier }}$ and $\Lambda_{\text {well }}$ and electron densities $\rho_{\text {barrier }}$ and $\rho_{\text {well }}$, respectively. It is worth mentioning that certain reflections may be suppressed in the diffraction patterns as a result of destructive interference between the sublayers when the thickness ratio $\Lambda_{\mathrm{MQW}} / \Lambda_{\mathrm{well}}$ is a rational number $m / n$, where $m$ and $n$ are integers. Therefore, the absence of the $4^{\text {th }}$-order satellite peak indicates that the MQW period is approximately four times the thickness of the well layer. The corresponding MQW period $\left(\Lambda_{\mathrm{MQW}}\right)$ derived from the satellite peak spacing, $\Delta q=0.2805 \mathrm{~nm}^{-1}$, is $\sim 22.4 \mathrm{~nm}$. The thicknesses $\Lambda_{\text {well }}$ and $\Lambda_{\text {barrier }}$ were estimated to be $\sim 5.4$ and $\sim 17.4 \mathrm{~nm}$, respectively.

In order to realize peak broadening and determine the overall strain state of the $\mathrm{Mg}_{0.03} \mathrm{Zn}_{0.97} \mathrm{O}$ buffer layer and the $\mathrm{ZnO} / \mathrm{ZnMgO} \mathrm{MQWs}$, reciprocal space mapping (RSM) near the asymmetric $\mathrm{Zn}_{0.97} \mathrm{Mg}_{0.03} \mathrm{O}(10 \overline{1} 5)$ reflection was performed and is shown in Fig. 2d. The unidentifiable (1015) reflections between $\mathrm{Zn}_{0.97} \mathrm{Mg}_{0.03} \mathrm{O}$ and the MQWs in the $\mathrm{H}-\mathrm{L}$ plane of the RSM indicate that the MQW structure is nearly fully strained; $\mathrm{ZnO} / \mathrm{ZnMgO} \mathrm{MQWs}$ were grown coherently on $\mathrm{Zn}_{0.97} \mathrm{Mg}_{0.03} \mathrm{O}$ buffer layers. Considering Fig. $2 \mathrm{c}$ and $\mathrm{d}$, the average lattice constants, $a$ and $c$, of the MQWs can be estimated to be 
approximately 0.3269 and $0.5174 \mathrm{~nm}$, respectively. In comparison to bulk $\mathrm{ZnO}$, with $a=0.3244$ and $c=0.5204 \mathrm{~nm}$, the lattice of the MQWs experienced tensile strain in the lateral direction of $\sim 0.77 \%$, but was compressed along the growth direction by approximately $-0.58 \%$.

To confirm the well width and interface structure between $\mathrm{Zn}_{0.8} \mathrm{Mg}_{0.2} \mathrm{O}$ and $\mathrm{ZnO}$ in the MQWs, a cross-sectional TEM measurement was performed. Fig. $3 \mathrm{a}$ and $\mathrm{b}$ show low and high magnification TEM micrographs, respectively, along the $[10 \overline{10}]_{\mathrm{Al}_{2} \mathrm{O}_{3}}$ projection. The TEM image also reveals good periodicity and a uniform distribution across the entire 10-pair MQW structure. The estimated period of the MQWs, consistent with the XRD results, is approximately $22.4 \mathrm{~nm}$. Moreover, the estimated thicknesses of the $\mathrm{ZnO}$ well and $\mathrm{Zn}_{0.8} \mathrm{Mg}_{0.2} \mathrm{O}$ barrier layer from Fig. $3 \mathrm{~b}$ are 5.4 and $17 \mathrm{~nm}$, respectively. These results again illustrate that the $4^{\text {th }}$ satellite peak in the XRD measurement (Fig. 2c) is suppressed because the ratio of $\Lambda_{\mathrm{MQW}}$ to $\Lambda_{\mathrm{well}}$ is approximately 4 . Fig. $3 \mathrm{c}$ shows the high-angle annular dark field (HAADF) STEM image, which is known to be highly sensitive to variation in atomic species. The contrast between each $\mathrm{ZnO}$ well layer is clearly enhanced, owing to the atomic-number dependence of the HAADF-STEM intensity, and homogeneous epitaxial growth is thereby observed together with the thickness of each ZnO layer. Furthermore, the buckled or wrinkled structure in the HAADF-STEM image is attributed to the larger lattice strain caused by unrelaxed growth of the $\mathrm{Zn}_{0.97} \mathrm{Mg}_{0.03} \mathrm{O}$ buffer layers.

The RT PL spectrum, as shown in Fig. 3d, was examined to characterize the optical performance of the MQWs. After fitting to a Gaussian profile, the near-band-edge emission of the
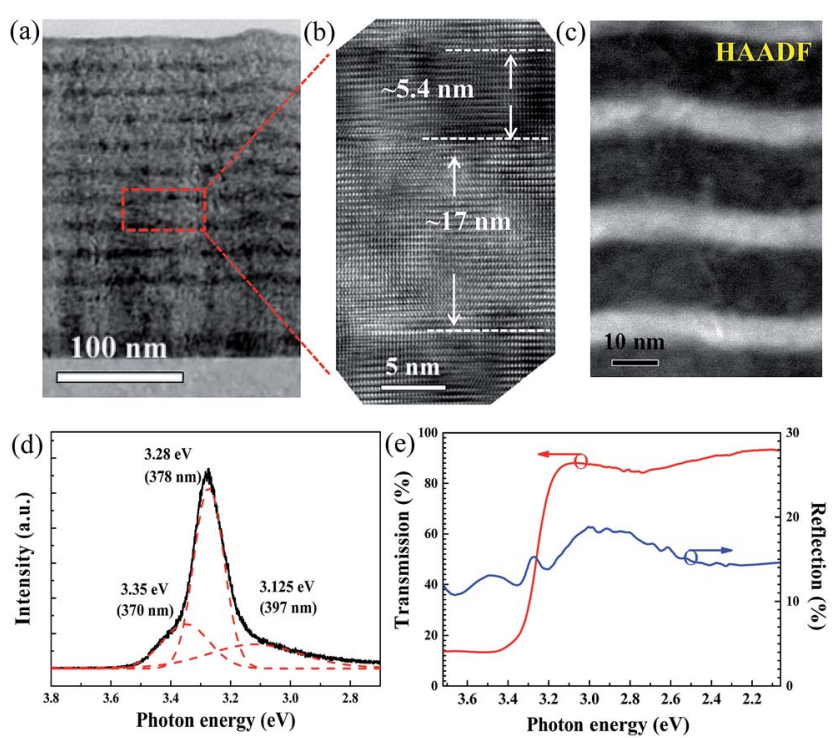

Fig. 3 (a) Cross-sectional TEM micrograph recorded along the $[1010]_{\mathrm{Al}_{2} \mathrm{O}_{3}}$ projection. (b) High-resolution image of the $\mathrm{ZnO} / \mathrm{ZnMgO}$ MQWs in the boxed region shown in (a). (c) Cross-sectional HAADFSTEM image. (d) PL spectra of the ten-pair $\mathrm{ZnO} / \mathrm{ZnMgO} \mathrm{MQW} \mathrm{film} \mathrm{on}$ (0001) sapphire measured at $300 \mathrm{~K}$. (e) RT transmittance (red solid curve) and reflectance (blue solid curve) spectra of the ten-pair $\mathrm{ZnO} /$ ZnMgO MQW film on (0001) sapphire.
MQWs can be clearly decomposed into three emission peaks, located at $397 \mathrm{~nm}, 378 \mathrm{~nm}$ and $370 \mathrm{~nm}$. The emission peaks at $378 \mathrm{~nm}(3.28 \mathrm{eV})$ and $370 \mathrm{~nm}(3.35 \mathrm{eV})$ are dominated by the free exciton (FX) emission of $\mathrm{ZnO}$ and $\mathrm{Zn}_{0.97} \mathrm{Mg}_{0.03} \mathrm{O}$, respectively. The other strong emission peak at $397 \mathrm{~nm}$, representing surface-bound-exciton emission, originates from the radiative recombination of excitons bound to interface defects in the $\mathrm{ZnO}$ layers. ${ }^{24}$ The photoluminescence of the barrier layer was not observed in the PL spectrum because the bandgap of $\mathrm{Zn}_{0.8} \mathrm{Mg}_{0.2} \mathrm{O}$ is larger than that of the $\mathrm{He}-\mathrm{Cd}$ pumping source $(3.815 \mathrm{eV})$. To further determine the excitonic recombination energy levels, the transmittance (red solid curve) and reflectance (blue solid curve) spectra were explored, as illustrated in Fig. 3e. The peak at $3.28 \mathrm{eV}$ (corresponding to $378 \mathrm{~nm}$ ) in the reflectance spectrum, consistent with the PL measurement results, is assigned to the $\mathrm{FX}$ of the $\mathrm{ZnO}$ well. Thus, considering the exciton binding energy of $\sim 60 \mathrm{meV}$, the bandgap of the $\mathrm{ZnO}$ layer is $\sim 3.34 \mathrm{eV}$ (corresponding to $371 \mathrm{~nm}$ ).

To investigate the behavior of CAP oscillations in $\mathrm{ZnO} /$ ZnMgO MQWs around the exciton resonance, TDT and TDR measurements were performed using the degenerate pumpprobe technique. Fig. $4 \mathrm{a}$ and b show the measured TDT $(\Delta T / T)$ and TDR $(\Delta R / R)$ traces, respectively. Measurements were taken with the excited photon energy of the pump pulse tuned above the exciton resonance $\left(3.28 \mathrm{eV}\right.$, pump wavelength $\left.\lambda_{\mathrm{p}}=378 \mathrm{~nm}\right)$, but below the bandgap of the $\mathrm{ZnO}$ layer $\left(3.34 \mathrm{eV}, \lambda_{\mathrm{p}}=371 \mathrm{~nm}\right)$. Except for $\lambda_{\mathrm{p}}$ at $371 \mathrm{~nm}$, the TDT and TDR signals generally start with a sharp dip around the zero time delay, owing to the twophoton absorption (TPA). The observation of TPA at a belowbandgap excitation of the $\mathrm{ZnO}$ well can be attributed to the absorption of a pump photon to create an exciton, followed by ionization and successful absorption of a probe photon. ${ }^{\mathbf{1 6}}$

Additionally, free electron-hole pairs can also be excited by cascade absorption of two pump photons via the exciton level. After a dip around the zero time delay, an instantaneous rise is observed in the TDT and TDR traces due to the occupation of the exciton level inhibiting the subsequent absorption of probe photons. This is termed the band-filling (BF) effect. Through carrier-phonon scattering thermalization, the excited free carriers gradually relaxed to low energy levels. Accumulated free carriers at the bottom of the band edges screened the Coulomb potential energy and resulted in shrinkage of the bandgap, termed the bandgap renormalization (BGR). This then caused the increase in absorption of probe photons and led to negative
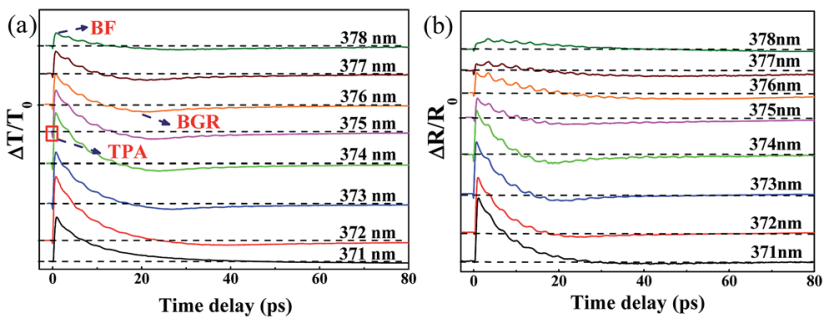

Fig. 4 (a) The TDT traces $(\Delta T / T)$ and (b) the TDR traces $(\Delta R / R)$ of $\mathrm{ZnO} /$ ZnMgO MQWs for pump wavelengths from $371 \mathrm{~nm}$ to $378 \mathrm{~nm}$. 
transient transmittance signals. On the other hand, as the pump photon energy was set near the band edge of $\mathrm{ZnO}$, i.e., $\lambda_{\mathrm{p}}=371 \mathrm{~nm}$, the photo-excited carriers occupied the band-edge states and prevented further absorption of probe photons. Therefore, only positive BF was detected in the TDT and TDR signals. In addition to the aforementioned ultrafast carrier dynamics of MQWs, obvious oscillations were superimposed on both the TDT and TDR traces. The oscillations arose from light scattering through the periodic distribution of the photoexcited carrier population induced by the $c$-axis MQW structure with the wave vector $q=2 \pi / \Lambda_{\mathrm{MQW}}$ which enabled the observation of coherent oscillations of zone-folded acoustic phonons.

The evolution of the TDT traces at $\lambda_{\mathrm{p}}=371 \mathrm{~nm}$ with increasing pump power $\left(P_{\mathrm{p}}\right)$ (from $4 \mathrm{~mW}$ to $16 \mathrm{~mW}$, or 4 to $16 \mu \mathrm{J}$ $\mathrm{cm}^{-2}$ ) at a fixed probe power (approximately $0.5 \mathrm{~mW}$, or $0.5 \mu \mathrm{J}$ $\mathrm{cm}^{-2}$ ) is shown in Fig. 5a. It is worth noting that no negative signal resulting from the BGR effect was observed in these traces. All the TDT traces exhibited a positive peak resulting from the BF effect, followed by a gradual decay that can be fitted well using the bi-exponential formula: ${ }^{15}$

$$
\frac{\Delta T}{T}=A_{1} \exp \left(-\frac{t}{\tau_{1}}\right)+A_{2} \exp \left(-\frac{t}{\tau_{2}}\right)
$$

where $A_{1}$ and $A_{2}$ are scaling factors and $\tau_{1}$ and $\tau_{2}$ represent fast and slow decay time constants of approximately 6 ps and $20 \mathrm{ps,}$ respectively. The time constant $\tau_{1}$ is responsible for the relaxation of free electrons to the bottom of the conduction band via electron-phonon scattering and $\tau_{2}$ is attributed to the nonradiative carrier recombination time. ${ }^{25}$ After subtraction of the fitted curve from the measured TDT trace at $P_{\mathrm{p}}=16 \mathrm{~mW}$, we obtained a damped oscillation signal with an amplitude of approximately $10^{-4}$ (Fig. 5b), which is attributed to the CAPs. After applying a fast Fourier transformation (FFT), the spectrum
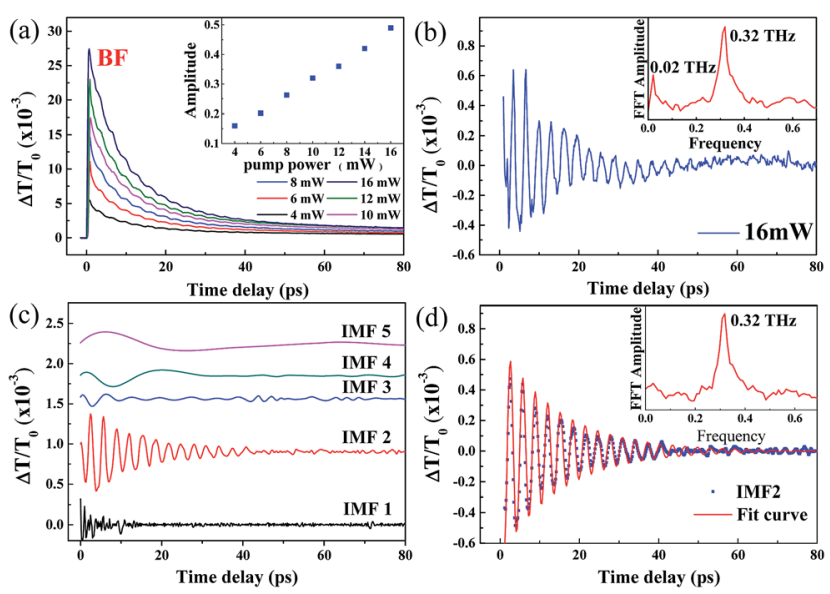

Fig. 5 (a) Pump-power-dependent TDT traces and CAP oscillation amplitude (inset) for a pump wavelength of $371 \mathrm{~nm}$. (b) The CAP oscillation extracted from the TDT trace with $P_{\mathrm{p}}$ at $16 \mathrm{~mW}$ and the corresponding FFT spectrum (inset). (c) The extracted intrinsic mode functions (IMF1 to IMF5) for the CAP oscillation and (d) IMF2 from the CAP oscillation and the fitting curve described by eqn (2) and the FFT spectrum (inset). (inset of Fig. 5b) indicates two distinctive oscillation frequencies at approximately $0.02 \mathrm{THz}$ and $0.32 \mathrm{THz}$. However, the damped oscillation was superimposed with noise and modulated by a low-frequency signal from the environment. In order to filter out these ambiguous effects we employed the empirical mode decomposition (EMD) method proposed by Huang et al. ${ }^{21,22}$ to analyze our signals. The decomposition is based on the assumption that each data set consists of different simple intrinsic mode functions (IMFs), the definition of which requires that (i) the number of extrema and zero-crossings in the whole data set must either be equal or differ at most by one and (ii) the mean values of the envelope defined by the local maxima and the envelope defined by the local minima are zero. Therefore, any complicated data set can be decomposed into a finite and often small number of IMFs that admit wellbehaved Hilbert transforms. This provides a new method for analyzing nonlinear and non-stationary data.

The decomposed IMFs (IMF1 to IMF5) from the damped oscillation signals of Fig. 5b are shown in Fig. 5c. The first IMF (IMF1) obviously results from the noise. The higher-order IMFs with low amplitude, such as IMF3 to IMF4, might contribute, but only slightly, to the CAP oscillation. IMF5, with a low oscillation frequency of approximately $0.02 \mathrm{THz}$, may result from the echo of the acoustic wave in the MQW configuration. Thus, the CAP oscillation mainly results from IMF2 (the blue dots in Fig. 5d), which can be fitted well using the damped oscillation formula:

$$
\frac{\Delta T}{T}=A_{\mathrm{c}} \cos \left(\frac{2 \pi t}{T_{\mathrm{p}}}\right) \exp \left(-\frac{t}{\tau}\right)
$$

where $A_{\mathrm{c}}=0.5, T_{\mathrm{p}}=0.32 \mathrm{ps}$ and $\tau=17 \mathrm{ps}$ are the amplitude, period and decay constant of the CAP oscillation, respectively. In addition, the oscillation amplitude $A_{\mathrm{c}}$ exhibits a linear increase with pump power after fitting, as shown in the inset of Fig. 5a. As seen previously in superlattices, ${ }^{6}$ the period of each pair inside the MQW structure $\left(\Lambda_{\mathrm{MQW}}\right)$ is given by the formula $\Lambda_{\mathrm{MQW}}=c_{\mathrm{SL}} \times T_{\mathrm{p}}$. As the velocity $c_{\mathrm{SL}}$ of longitudinal acoustic waves inside $\mathrm{ZnO}^{26}$ is approximately $6400 \mathrm{~m} \mathrm{~s}^{-1}$ and the oscillation period $T_{\mathrm{p}}$ is $0.32 \mathrm{ps}$, the estimated period of the MQWs is approximately $21 \mathrm{~nm}$, close to the value of $22.4 \mathrm{~nm}$ measured with XRD and TEM. Thus, the oscillation from the $\mathrm{ZnO} / \mathrm{ZnMgO}$ MQWs in our pump-probe measurement can be attributed to the zone-folded acoustic phonon mode.

The TDT traces of $\mathrm{ZnO} / \mathrm{ZnMgO}$ MQWs at various pump powers $\left(P_{\mathrm{p}}=2\right.$ to $\left.18 \mathrm{~mW}\right)$ with the excited photon energy set near the exciton resonance of the $\mathrm{ZnO}$ well layer at $3.31 \mathrm{eV}\left(\lambda_{\mathrm{p}}=\right.$ $375 \mathrm{~nm})$ are shown in Fig. $6 \mathrm{a}-\mathrm{d}$. At a lower pump power $\left(P_{\mathrm{p}}=2\right.$ $\mathrm{mW}$ ), the TDT trace in Fig. 6 a reveals only a positive peak caused by the BF effect, and then gradually relaxes due to carrierphonon scattering. As the pump power is increased above 4 $\mathrm{mW}$, the TPA dip and the negative $\left|\Delta T / T_{0}\right|_{\mathrm{BGR}}$ value due to the BGR effect can both be observed in the TDT traces (Fig. 6b-d). As we further increase the pump power, the maximum modulation depths of the TDT traces, such as $\left|\Delta T / T_{0}\right|_{\text {TPA }}$ (labeled in Fig. 6c), caused by the TPA at the zero time delay and $\left|\Delta T / T_{0}\right|_{\text {BGR }}$ (labeled in Fig. 6d), caused by the BGR at a certain time delay 

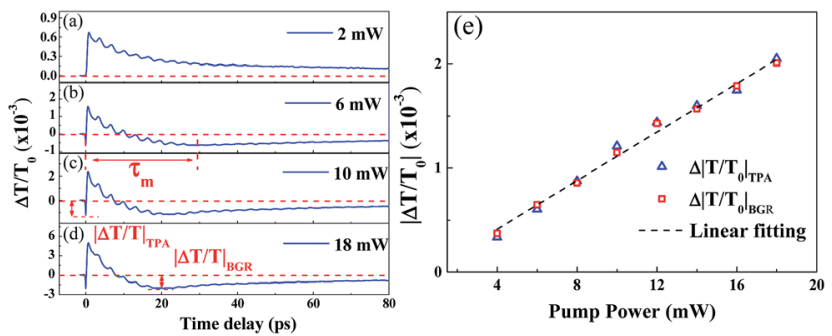

Fig. 6 (a-d) TDT traces for pump power increasing from $2 \mathrm{~mW}$ to 18 $\mathrm{mW}$ for a pump wavelength of $375 \mathrm{~nm}$. (e) Modulation depths of TPA $\left(\left|\Delta T / T_{0}\right|_{\text {TPA }}\right)$ and BGR $\left(\left|\Delta T / T_{0}\right|_{B G R}\right)$ as a function of pump power.

( $\tau_{\mathrm{m}}$, the BGR buildup time), become obvious. Moreover, $\tau_{\mathrm{m}}$ decreases as the pump power increases.

The TPA effect around the exciton resonance can be reasonably explained by the cascaded absorption of two pump photons or the simultaneous absorption of one pump and one probe pulse around the zero time delay, exciting the electron from the valence band (VB) to the exciton level and then to the conduction band (CB). ${ }^{15,16}$ This is termed the resonance TPA. Unlike non-resonance TPA, caused by the virtual transition and exhibiting a nonlinear relation with $P_{\mathrm{p}}$, the modulation depths of TPA $\left(\left|\Delta T / T_{0}\right|_{\text {TPA }}\right)$ around the zero time delay and BGR $(|\Delta T|$ $\left.\left.T_{0}\right|_{\text {BGR }}\right)$ at $\tau_{\mathrm{m}}$ increase linearly with the pump power and are consistent with each other, as shown in Fig. 6e. For BGR, most free electrons can be excited via the exciton level to the $\mathrm{CB}$, leaving holes in the VB, by means of the cascaded absorption of two pump photons. Through carrier-phonon scattering, the cooled carriers occupy the lower lying electronic states at the bottom of the CB and/or the hole states at the top of the VB, as has been previously reported in polar $\mathrm{ZnO} .^{16} \mathrm{Then}$, the Coulomb potential energy is screened, resulting in the BGR effect. This is the reason why the modulation depth $\left|\Delta T / T_{0}\right|_{\text {BGR }}$ is proportional to $\left|\Delta T / T_{0}\right|_{\text {TPA. }}$ As pump power increases, more free electrons are excited to the $\mathrm{CB}$ at an earlier time, speeding up electron-electron and electron-phonon scattering. Similar processes also occur for the excited holes in the VB. Thus, the free carriers relax quickly to the band edge and cause the BGR to occur early at the higher pumping powers.

Fig. 7a-d show the retrieved IMFs of CAP oscillations from the TDT traces in Fig. $6 \mathrm{a}-\mathrm{d}$. The blue dots in Fig. 7a reveal a monotonically damped IMF CAP oscillation at $P_{\mathrm{p}}=2 \mathrm{~mW}$. The IMF mode can be fitted well using eqn (2) (red line) with a single oscillation frequency of approximately $0.32 \mathrm{THz}$, as shown in the FFT spectrum (inset of Fig. 7a). The IMFs retrieved when the pump power was increased from $6 \mathrm{~mW}$ to $18 \mathrm{~mW}$ are shown by the blue dots in Fig. 7b-d. In contrast to Fig. 7a, the beating of two distinct oscillation signals is shown in these plots. Thus, we fitted the damped traces with a formula containing a superposition of two oscillation signals:

$$
\frac{\Delta T}{T}=A_{\mathrm{c}}\left[\cos \left(\frac{2 \pi t}{T_{\mathrm{p} 1}}\right)+\cos \left(\frac{2 \pi t}{T_{\mathrm{p} 2}}\right)\right] \exp \left(-\frac{t}{\tau}\right)
$$

where $A_{\mathrm{c}}$ is a scaling factor, $T_{\mathrm{p} 1}$ and $T_{\mathrm{p} 2}$ are the periods of the two oscillation signals and $\tau$ is a decay constant. The well-fitted
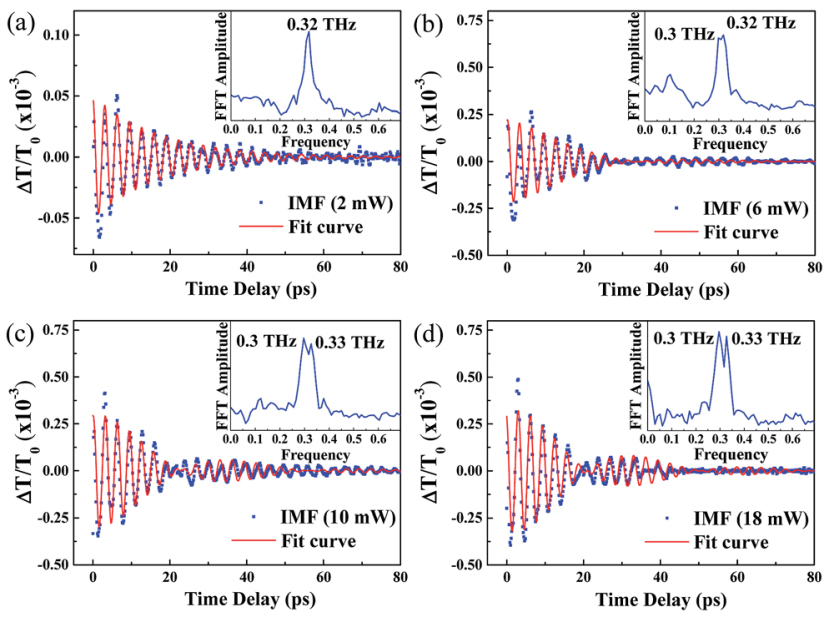

Fig. 7 The CAP oscillation extracted from the TDT trace for a pump wavelength of $375 \mathrm{~nm}$ and pump power of (a) $2 \mathrm{~mW}$, (b) $6 \mathrm{~mW}$, (c) 10 $\mathrm{mW}$ and (d) $18 \mathrm{~mW}$.

curves are shown in red in Fig. 7b-d. The corresponding FFT spectra (the insets) reveal that the amplitude of the additional oscillation, with a frequency of approximately $0.3 \mathrm{THz}$, increased with pump power. The occurrence of the additional oscillation frequency should correlate with the BGR which produces the negative TDT traces, as shown in Fig. 6b-d. Theoretically, the induced refractive index change $(\Delta n)$ from the BGR is negative $(\Delta n<0)$, opposite to the BF effect with $\Delta n>0$. Therefore, based on Thomsen's model, ${ }^{27}$ the oscillation frequency of CAP can be expressed by the formula $\nu=\left(2 n c_{\mathrm{SL}}\right) / \lambda_{\mathrm{p}}$. When $\Delta n<0, n$ decreases to cause a reduction in the oscillation frequency. In addition, the amplitude of the low frequency peak at $0.3 \mathrm{THz}$ is slightly larger than that of the peak at $0.32 \mathrm{THz}$ because of the longer dwell time in the BGR state than in the BF state, as shown in Fig. 6c and d.

Fig. 8 shows the amplitude of CAP oscillation as a function of pump power or excited carrier density $\left(N_{\text {exc }}\right)$. Here, the excited carrier density is estimated using the formula $N_{\text {exc }}=I_{\text {exc }} \alpha /$ $\hbar \omega_{\text {exc }},{ }^{22,25}$ where $I_{\text {exc }}$ is the pumping fluence (or energy flux density), $\alpha$ is the absorption coefficient and $\hbar \omega_{\text {exc }}$ is the photon

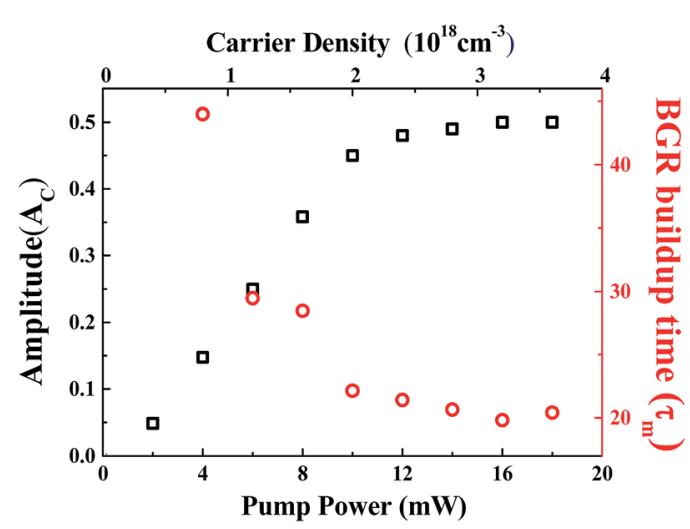

Fig. 8 The amplitude of CAP oscillations $A_{c}$ and BGR buildup time $\left(\tau_{m}\right)$ as a function of pump power. 
energy. The absorption coefficient $\alpha$ can be obtained from the transmittance $T_{0}$ (Fig. 3e) by the formula: ${ }^{28}$

$$
T_{0}=\frac{\left(1-R_{1}\right)\left(1-R_{2}\right) \exp (-\alpha D)}{1-R_{1} R_{2} \exp (-2 \alpha D)}
$$

where $R_{1}$ is the reflectance between air and the $\mathrm{ZnO}, R_{2}$ is the reflectance between the $\mathrm{ZnO}$ and the sapphire and $D$ is the thickness of the well. From Fig. 3e, the obtained value for $\alpha$ is approximately $1.12 \times 10^{5} \mathrm{~cm}^{-1}$ at a pump wavelength of $375 \mathrm{~nm}$. The diameters of the focused pump and probe beams were measured to be $42-45 \mu \mathrm{m}$. In contrast to the linear dependence of the modulation depth of TPA and BGR on the pump power (Fig. 6e), the amplitude $A_{\mathrm{c}}$ of CAP oscillation (Fig. 8) increases almost linearly with pump power $P_{\mathrm{p}}$ at lower excited carrier densities, but saturates as $P_{\mathrm{p}}$ increases beyond 10 $\mathrm{mW}$.

By analyzing the initial phase of CAP oscillation near the exciton resonance and bandgap, as shown in Fig. 5 and 7, the $\cos \left(\omega_{\text {phonon }} t\right)$ dependence was observed. Based on the mechanism of coherent phonon generation ${ }^{29,30}$ the CAP oscillations of the $\mathrm{ZnO}$ well layers in the polar $\mathrm{ZnO} / \mathrm{ZnMgO}$ MQWs are induced via DECP. Theoretically, the driving force of a harmonic oscillator can be expressed by the sum of the Raman term and the nonlinear longitudinal polarization. ${ }^{29,30}$ The nonlinear polarization can be attributed to the second- and the third-order nonlinear susceptibilities as well as a longitudinal polarization generated by ultrafast drift-diffusion currents, which can excite coherent phonons via transient depletion field screening (TDFS), and the polarization associated with coherent electronic wavefunctions. ${ }^{29,30}$

Generally, more carriers are excited at higher pump powers that perturb the built-in electric field, enhancing the amplitude of CAP. Nevertheless, the built-in electric field should be screened by the photo-induced carriers to decrease ultrafast drift-diffusion currents via TDFS, ${ }^{31}$ i.e., reducing the band-tilted effect. Therefore, when the screening of the built-in electric field by photon-induced carriers is carried out at a higher excited carrier density for polar $\mathrm{ZnO} / \mathrm{ZnMgO} \mathrm{MQWs}$, it will cause the decline of nonlinear polarization and reduce the driving force of harmonic oscillation. Hence the saturation of the CAP amplitude is observable. Fig. 8 also shows the BGR build-up time $\tau_{\mathrm{m}}$, the relative time delay at the TDT trace minimum, as a function of excited carrier density. It reveals that $\tau_{\mathrm{m}}$ decreases linearly with increasing carrier density and levels off as the carrier density increases beyond $2 \times 10^{18} \mathrm{~cm}^{-3}$ (or $P_{\mathrm{p}}=10 \mathrm{~mW}$ ) through the cascade TPA absorption, i.e., the internal electric field would be fully screened when the number of excited carriers is beyond $2 \times 10^{18} \mathrm{~cm}^{-3}$. Like the aforementioned results in Fig. 1b and c, the periodic distribution of excited carriers from the TPA produces electronic strain that couples with the selective zone-folded acoustic-phonon modes via DECP. With further increases in the pump power, more free carriers are excited and the amplitude of CAP oscillation is enhanced. On the other hand, the higher carrier density causes fast cooling of the high-lying carriers to the band edge via carrier-carrier scattering and carrier-phonon scattering, leading to the fast build-up of BGR. At a relatively high carrier density the built-in field might also be screened, flattening the potential well of the MQW structure and suppressing the increase in $A_{\mathrm{c}}$. Therefore, the flattening of the potential well, rather than the carrier density, is responsible for the saturation of $A_{\mathrm{c}}$ and $\tau_{\mathrm{m}}$. The carrier density accounts for the linear dependence of BGR modulation depth on the pump power in Fig. 6e.

\section{Conclusions}

In this work, we grew $\mathrm{ZnO} / \mathrm{Zn}_{0.8} \mathrm{Mg}_{0.2} \mathrm{O}$ multiple quantum wells (MQWs) with high structural quality and regularly arranged well and barrier layers using pulsed laser deposition, as evidenced by high-resolution cross-sectional transmission electron microscopy (TEM) images and the pronounced high-order satellite peaks in X-ray crystal truncation rods. The thicknesses of the well and barrier layers can be estimated from the satellite peak spacing and the suppressed intensity of the $4^{\text {th }}$ order satellite peak in the X-ray diffraction (XRD) pattern. They are approximately 5.4 and $17.4 \mathrm{~nm}$, respectively, and are consistent with the TEM image. When the excited photon energy was set between the band edge and the exciton resonance, sub-THz coherent acoustic phonon (CAP) oscillation from the $\mathrm{ZnO} / \mathrm{Mg}_{0.2} \mathrm{Zn}_{0.8} \mathrm{O}$ MQWs was demonstrated in a degenerate pump-probe measurement. The occurrence of acoustic waves is mainly attributed to the perturbation of the internal electric field resulting from photo-excited carriers based on cascade two-photon absorption. By applying empirical mode decomposition, the purified signal can be retrieved to calculate the CAP oscillation frequency of $\sim 0.32 \mathrm{THz}$ and the MQW period of $\sim 21 \mathrm{~nm}$, consistent with the XRD and TEM results. With the increase in photo-excited power (or excited carriers), an additional oscillation frequency of the acoustic wave, $0.3 \mathrm{THz}$, was observed because the bandgap renormalization effect caused a negative change in refractive index $(\Delta n<$ 0 ) in the MQWs. Furthermore, the saturation of CAP amplitude at high pumping power is attributed to the screening of the built-in electric field by the generation of many photo-excited carriers.

\section{Conflicts of interest}

There are no conflicts to declare.

\section{Acknowledgements}

This work was supported by the Ministry of Science and Technology of Taiwan, under grants MOST 105-2112-M-213-008 and MOST 105-2112-M-027-001-MY3.

\section{Notes and references}

1 B. C. Daly, N. C. R. Holme, T. Buma, C. Branciard, T. B. Norris, D. M. Tennant, J. A. Taylor, J. E. Bower and S. Pau, Appl. Phys. Lett., 2004, 84, 5180-5182. 
2 K. H. Lin, C. T. Yu, S. Z. Sun, H. P. Chen, C. C. Pan, J. I. Chyi, S. W. Huang, P. C. Li and C. K. Sun, Appl. Phys. Lett., 2006, 89, 043106.

3 H. J. Zeiger, J. Vidal, T. K. Cheng, E. P. Ippen, G. Dresselhaus and M. S. Dresselhaus, Phys. Rev. B: Condens. Matter Mater. Phys., 1992, 45, 768-778.

4 R. Merlin, Solid State Commun., 1997, 102, 207-220.

5 G. A. Garret, T. F. Albrecht, J. F. Whitaker and R. Merlin, Phys. Rev. Lett., 1996, 77, 3661-3664.

6 P. Ruello and V. E. Gusev, Ultrasonics, 2015, 56, 21-35.

7 R. N. Kini, A. J. Kent, N. M. Stanton and M. Henini, Appl. Phys. Lett., 2006, 88, 134112.

8 O. B. Wright, B. Perrin, O. Matsuda and V. E. Gusev, Phys. Rev. B, 2001, 64, 081202.

9 P. Babilotte, P. Ruello, D. Mounier, T. Pezeril, G. Vaudel, M. Edely, J.-M. Breteau, V. Gusev and K. Blary, Phys. Rev. B: Condens. Matter Mater. Phys., 2010, 81, 245207.

10 E. S. K. Young, A. V. Akimov, R. P. Campion, A. J. Kent and V. Gusev, Phys. Rev. B: Condens. Matter Mater. Phys., 2013, 86, 155207.

11 C. K. Sun, J. C. Liang and X. Y. Yu, Phys. Rev. Lett., 2000, 84, 179-182.

12 G. D. Sanders, C. J. Stanton and C. S. Kim, Phys. Rev. B: Condens. Matter Mater. Phys., 2001, 64, 235316.

13 J. H. Lim, C. K. Kang, K. K. Kim, I. K. Park, D. K. Hwang and S. J. Park, Adv. Mater., 2006, 18, 2720-2724.

14 T. C. Lu, Y. Y. Lai, Y. P. Lan, S. W. Huang, J. R. Chen, Y. C. Wu, W. F. Hsieh and H. Deng, Opt. Express, 2012, 20, 5530-5537.

15 J. H. Lin, H. J. Su, C. H. Lu, C. P. Chang, W. R. Liu and W. F. Hsieh, Appl. Phys. Lett., 2015, 107, 142107.

16 J. H. Lin, W. R. Liu, Y. C. Lin, H. J. Su, H. R. Chen, C. Y. Tsai, Y. H. Chen and W. F. Hsieh, AIP Adv., 2016, 6, 095222.

17 C. K. Sun, S. Z. Sun, K. H. Lin, K. Y. J. Zhang, H. L. Liu, S. C. Liu and J. J. Wu, Appl. Phys. Lett., 2005, 87, 023106.
18 P. C. Ou, J. H. Lin, C. A. Chang, W. R. Liu and W. F. Hsieh, J. Phys. D: Appl. Phys., 2010, 43, 495103.

19 J. H. Lin, Y. K. Shen, W. R. Liu, C. H. Lu, Y. H. Chen, C. P. Chang, W. C. Lee, M. Hong, J. R. Kwo, C. H. Hsu and W. F. Hsieh, J. Phys. D: Appl. Phys., 2016, 49, 325102.

20 J. A. Davis and C. Jagadish, Laser Photonics Rev., 2009, 3, 8596.

21 N. E. Huang, Z. Shen, S. R. Long, M. C. Wu, H. H. Shih, Q. Zheng, N. Yen, C. C. Tung and H. H. Liu, Proc. R. Soc. London, Ser. A, 1998, 454, 903-995.

22 N. E. Huang, S. R. Long and Z. Shen, Adv. Appl. Mech., 1996, 32, 59-111.

23 P. Willmott, An Introduction to Synchrotron: Techniques and Application, Wiley, New York, 2011.

24 C. C. Kuo, B. H. Lin, S. Yang, W. R. Liu, W. F. Hsieh and C.-H. Hsu, Appl. Phys. Lett., 2012, 101, 011901.

25 P. C. Ou, W. R. Liu, H. J. Ton, J. H. Lin and W. F. Hsieh, J. Appl. Phys., 2011, 109, 013102.

26 Y. Lu, N. W. Emanetoglu and Y. Chen, in Zinc Oxide Bulk, Thin Films and Nanostructures, ed. C. Jagadish and S. J. Pearton, Elsevier, New York, 2006, ch. 13, pp. 443-489.

27 C. Thomsen, H. T. Grahn, H. J. Maris and J. Tauc, Phys. Rev. B: Condens. Matter Mater. Phys., 1986, 34, 4129-4138.

28 J. Ahmad, H. Minami, S. Alam, J. Yu, Y. Arai and H. Uwe, Chin. Phys. Lett., 2008, 25, 4421-4424.

29 K. Ishioka and O. V. Misochko, in Progress in Ultrafast Intense Laser Science, ed. A. Giulietti, and K. Ledingham, SpringerVerlag, Berlin Heidelberg, 2010, vol. V, ch. 2, pp. 23-46.

30 T. Dekorsy, G. C. Cho, and H. Kurz, in Light Scattering in Solids VIII, ed. M. Cardona and G. Güntherodt, SpringerVerlag, Berlin Heidelberg, 2001, vol. 76, ch. 4, pp. 169-209.

31 T. Dekorsy, T. Pfeifer, W. Kütt and H. Kurz, Phys. Rev. B: Condens. Matter Mater. Phys., 1993, 47, 3842-3849. 